\title{
Efeito da mosca-dos-chifres, Haematobia irritans (L.) (Diptera: Muscidae), no ganho de peso de bovinos Nelore
}

\author{
Effects of the horn fly, Haematobia irritans (L.) (Diptera: Muscidae) \\ in the weight gain on Nellore cattle
}

\author{
Ivo Bianchin ${ }^{1}$ Wilson Werner Koller ${ }^{2}$ Rafael Geraldo de Oliveira Alves ${ }^{3}$ \\ Edenio Detmann ${ }^{4}$
}

\section{RESUMO}

A presente investigação foi realizada devido à escassez, no Brasil, de informações sobre os possíveis danos decorrentes da ação hematófaga da mosca-dos-chifres, Haematobia irritans (L.), em bovinos da raça Nelore. O estudo foi realizado durante quatro estações chuvosas (outubro a abril), de 1991 a 1995. Em cada ano, 80 bovinos foram utilizados de acordo com os seguintes tratamentos: 28 touros com um ano de idade divididos em quatro grupos; 20 bois com idade de dois anos divididos em quatro grupos, e 32 bois com três anos e divididos em oito grupos. Metade dos grupos de animais de cada idade eram tratados contra $\boldsymbol{H}$. irritans com intervalos de 28 dias e os demais mantidos como grupos controle. As moscas foram contadas a cada 14 dias e o peso dos bois registrado a cada 28. O número médio de mosca/animal dos grupos controle para o primeiro, segundo, terceiro e quarto anos do estudo foi, respectivamente: cinco, cinco, quatro e cinco, nos animais de um ano; 15, 11, 13 e 27, nos de dois anos e 55, 31, 40 e 51, nos de três anos. Observou-se que maior número de moscas $(P<0,05)$ atacou os animais mais velhos. Houve, em todos os períodos de avaliação, apesar do número de moscas por animal poder ser considerado baixo, um ganho de peso significativo $(P<0,05)$ em prol dos grupos tratados contra $\boldsymbol{H}$. irritans comparativamente aos grupos não tratados. Os ganhos foram 8,$6 ; 16,0$ e 10,4\% superiores, respectivamente, para animais de um, dois e três anos de idade. Esse ganho de peso por animal e por ano foi da ordem de 9, 5, 7 e $8 \mathrm{~kg}$ para os animais com um ano de idade; de 26, 10, 23 e $12 \mathrm{~kg}$ para aqueles de dois anos, e de 16, 8, 9 e $11 \mathrm{~kg}$ nos de três anos.

Palavras-chave: bovinos, Nelore, mosca-dos-chifres, dano econômico, Brasil.

\section{ABSTRACT}

This investigation was carried out due the lack of information about the possible effects of the blood-feeding horn

\begin{abstract}
fly, Haematobia irritans (L.), on Nellore cattle. Data were recorded during four rainy seasons (October to April) from 1991 to 1995. In each year, 80 animals were utilized according to the following treatments: twenty-eight 1-year old bulls divided into four groups; twenty 2-year old cattle divided into four groups and thirty-two 3year old cattle divided into eight groups. Treatments against $\boldsymbol{H}$. irritans were conduced at about 28-day intervals on half part of each age groups and the remainders groups maintained without treatment as control. The flies were counted at 14-day intervals and the animals weight registered at each 28-day intervals. The values for the mean flies number on the animals of the control groups in the first, second, third and fourth years of this study were: five, five, four and five, in the 1-year old group; 15, 11, 13 and 27, in the 2-year old group, and 55, 31, 40 and 51, in that 3year old group, respectively. It was observed that higher number of flies $(P<0.05)$ attack adult animals than younger ones. Despite of the low number of flies per cattle, there was a significant weight gain $(P<0.05)$ in favour of treated groups than the controls, which gained 8.6, 16.0 and $10.4 \%$ over the period, for groups one, two and three years old, respectively. The weight gain per year was 9, 5,7 and $8 \mathrm{~kg}$ for the 1-year old groups; 26, 10, $23 \mathrm{~kg}$ and 12 in 2year old groups, and 16, 8, 9 and $11 \mathrm{~kg}$ in 3-year old groups.
\end{abstract}

Key words: cattle, Nellore, horn fly, economic damage, Brazil.

\section{INTRODUÇÃO}

Nos EUA, entre as 50 espécies de ecto e endoparasitos que acometem o rebanho bovino do país a mosca-dos-chifres, Haematobia irritans (L.), é considerada a que causa os maiores prejuízos (BYFORD et al., 1992), sendo que os prejuízos atribuídos a esta espécie foram estimados, já há cerca de uma década, em US\$ 876 milhões (KUNZ et al.,

\footnotetext{
${ }^{1}$ Médico Veterinário, DSc., Embrapa Gado de Corte, CP 154, 79002-970, Campo Grande, MS. E-mail: bianchin@cnpgc.embrapa.br. Autor para correspondência.

${ }^{2}$ Biólogo, DSc., Embrapa Gado de Corte.

${ }^{3}$ Engenheiro Agrônomo, DSc., Embrapa Gado de Corte.

${ }^{4}$ Zootecnista, DSc., Professor Visitante Departamento de Produção Animal, Núcleo de Ciências Veterinárias, Universidade Federal de Mato Grosso do Sul.
} 
1991). No Brasil, os danos atribuídos à mosca-doschifres com respeito ao rebanho nacional de bovinos foi estimada em US\$ 150 milhões (GRISI et al., 2002).

A relação parasito hospedeiro, assim como acontece, entre outros, com o carrapato Boophilus microplus (Canestrini), conforme relatado por UTECH (1978), tem relação direta com a espécie e/ ou a raça bovina considerada. Os bovinos são tanto mais suscetíveis aos parasitos quanto maior for sua taxa de sangue Bos taurus (raças de procedência européia). SCHREIBER et al. (1987), HOGSETTE et al. (1991) e MORRISON \& FOIL (1995) não verificaram diferenças no ganho de peso em vacas e ou bezerros pertencentes a raças $B$. taurus puras ou cruzadas infestados com $\boldsymbol{H}$. irritans, em relação aos animais nos quais essas moscas eram regularmente controladas. Por outro lado, STEELMAN et al. (1991) verificaram que animais da raça Chianina apresentaram metade da densidade populacional de moscas em comparação às raças Angus, Hereford, Polled Hereford, Red Poll e Charolês. Concluíram também que cada 100 moscas encontradas nas vacas de qualquer uma das raças estudadas corresponderam a uma perda de $8,1 \mathrm{~kg}$ de ganho de peso nos respectivos bezerros, sendo que vacas com menor número de moscas produziram bezerros mais pesados do que aquelas mais intensamente infestadas. COCKE et al. (1989) também observaram ganho de peso significativo em animais que tiveram as moscas controladas, quando comparados com animais infestados da respectiva raça, bem como diferenças entre raças, sendo que os animais da raça Simental apresentaram o maior ganho de peso verificado.

Com base nos resultados acima, BYFORD et al. (1992) concluíram que, mesmo entre raças de $\boldsymbol{B}$. taurus e/ou animais resultantes de cruzamentos com participação de B. taurus, ocorrem diferentes respostas à ação do parasito em questão, variando de danos nulos até economicamente importantes. Isso reforça a lógica de que cada caso merece um estudo à parte. Assim sendo, apesar de animais da raça Nelore (Bos indicus) terem apresentado menor infestação de parasitos como o carrapato B. microplus do que animais oriundos de raças européias ou de seus cruzamentos (UTECH et al., 1978; GOMES et al., 1989), os possíveis danos que a mosca-dos-chifres pode ocasionar (ou não) ao rebanho Nelore do país precisam ainda ser devidamente investigados.

A importância da raça Nelore para a pecuária nacional é relatada por SANTOS (1998), com as seguintes palavras: "Pode-se afirmar que $65,0 \%$ dos bovinos de corte do Brasil são anelorados, ou tem influência de sangue Nelore, ou seja, este rebanho de quase 100 milhões de cabeças é o maior do mundo".

A literatura sobre a dinâmica populacional da mosca-dos-chifres para a região do Brasil Central resume-se, na atualidade, a quatro estudos. Dois foram realizados no Estado de Mato Grosso do Sul: BARROS (2001), em Corumbá e BIANCHIN \& ALVES (2002), em Campo Grande; e os demais foram feitos no Estado de São Paulo: OLIVEIRA \& FREITAS (1997), em São Carlos, e LIMA et al. (2002), em Araçatuba. Apenas o relato de BIANCHIN \& ALVES (2002) inclui a determinação dos danos causados por este parasito em animais da raça Nelore, mas foi direcionado apenas para vacas e respectivo bezerro até o desmame. As demais informações existentes no país sobre este assunto são ainda de caráter preliminar, principalmente resumos em congressos.

De modo geral, as condições em que foram realizados em outros países os estudos existentes sobre o assunto aqui abordado são muito diferentes das existentes no Brasil. No Brasil, dada a grande extensão territorial ocupada pela pecuária bovina, os estudos devem levar em consideração suas diferentes regiões fisiográficas com as respectivas peculiaridades edafoclimáticas; os diferentes tipos de exploração pecuária: bovinos de corte, bovinos para leite e/ou de aptidão mista - corte e leite; as diferentes raças e ou cruzamentos em uso e os diferentes sistemas de criação e/ou de manejo utilizados: extensivos, semi-intensivos, intensivo ou confinamentos. Daí resultam múltiplas e distintas interações possíveis da relação "parasito $\mathrm{x}$ hospedeiro x ambiente".

O presente estudo teve por objetivos melhorar os conhecimentos sobre o efeito da moscados-chifres no ganho de peso em machos da raça Nelore, dada a importância dessa raça em termos numéricos no rebanho nacional de gado de corte.

\section{MATERIAL E MÉTODOS}

O trabalho foi desenvolvido na área experimental do Centro Nacional de Pesquisa de Gado de Corte, da Empresa Brasileira de Pesquisa Agropecuária, em Campo Grande, MS, localizado a $20^{\circ} 27^{\prime} \mathrm{S}$ e $54^{\circ} 37^{\prime} \mathrm{W}$ e a uma altitude aproximada de 530 metros. Segundo a classificação de KÖPPEN (1928), situa-se na faixa de transição entre o clima Cfa mesotérmico úmido sem estiagem e o clima AW tropical úmido, com estação chuvosa no verão e seca no inverno. A temperatura média do mês mais quente é superior a $22^{\circ} \mathrm{C}$, apresentando no mês mais seco mais de $30 \mathrm{~mm}$ de precipitação. De modo geral, o clima no 
Brasil Central caracteriza-se por apresentar uma estação seca de maio a setembro, e uma estação chuvosa, nos meses de outubro a abril.

O estudo foi realizado durante a estação chuvosa (outubro a maio), época de maior infestação de $\boldsymbol{H}$. irritans nos animais, em quatro períodos de infestação consecutivos: 1991/92; 1992/93; 1993/94 e 1994/95.

Foram utilizados 80 machos, que eram substituídos por um novo lote de animais ao final de cada período de infestação. Destes animais, 28 eram tourinhos de um ano de idade (A), 20 eram de dois anos e castrados (B), e 32, de três anos e castrados (C). Os animais foram agrupados por peso em números pares de grupos, por idade, de modo que a soma do peso vivo dos animais de cada grupo, no início de cada período de estudo, não diferissem entre si (Tabela 1). O arranjo acima se fez necessário para que se pudesse trabalhar com número total de animais por idade relativamente próximos, visto que os piquetes utilizados eram de mesmo tamanho. Dessa forma resultaram quatro grupos de sete animais com um ano de idade; quatro grupos de cinco animais com dois anos de idade e oito grupos de quatro animais com três anos de idade.

Os animais de metade dos grupos, de cada uma das idades em estudo (um, dois e três anos), foram tratados a cada 28 dias com inseticida do grupo Piretróide, princípio ativo Deltametrina, recomendado para o controle de $\boldsymbol{H}$. irritans, seguindo-se as recomendações do fabricante quanto ao método de aplicação e dosagem, de modo a mantê-los livres do parasito. Assim sendo, foram utilizados dois tratamentos (animais tratados com inseticidas $\mathrm{x}$ animais não tratados = grupo "controle") e três idades de animais, acima mencionadas. Para a análise dos resultados cada animal representou uma repetição. A razão de formar grupos com diferentes números de animais, dentro de cada idade, está relacionada à oferta de alimento (peso vivo dos animais por área de pastagem do piquete). Conforme acima mencionado, os piquetes eram todos do mesmo tamanho (quatro hectares), formados com Brachiaria brizantha. A taxa de lotação média no início de cada período de observação foi de 0,65 UA/ha, alcançando $1 \mathrm{UA} / \mathrm{ha}$ no final de cada período observado. Considerou-se a $\mathrm{UA} / \mathrm{ha}$, ou Unidade Animal por hectare, como correspondendo à $\pm 450 \mathrm{~kg}$ peso vivo.

No início do experimento e, a partir daí, a cada 14 dias, imobilizando-se os animais no tronco, foram realizadas, diretamente sobre todos os animais em estudo, contagens do número de mosca-dos-chifres em um dos lados e o resultado multiplicado por dois (BIANCHIN \& ALVES, 2002). Segundo a metodologia empregada por estes mesmos autores, foi também efetuada, a cada 28 dias, a pesagem dos animais. Essas pesagens foram realizadas durante $o$ período de maior incidência de $\boldsymbol{H}$. irritans na região de estudo, que é de novembro a abril (BIANCHIN \& ALVES, 2002).

As análises estatísticas foram conduzidas utilizando-se o programa do SAS Institute (1998),

Tabela 1 - Peso inicial (I) e final (F) em quilogramas de bovinos da raça Nelore, machos, com diferentes anos de idade (Id = 1, 2 e 3 anos); em função dos tratamentos (Tr) "com" e "sem" inseticidas, durante quatro estações chuvosas (outubro a maio) consecutivas, de 1991 a 1995, em Campo Grande, MS.

\begin{tabular}{|c|c|c|c|c|c|c|c|c|c|}
\hline \multirow{2}{*}{ Id } & \multirow{2}{*}{$\operatorname{Tr}$} & \multicolumn{2}{|c|}{$1991 / 92$} & \multicolumn{2}{|c|}{$1992 / 93$} & \multicolumn{2}{|c|}{$1993 / 94$} & \multicolumn{2}{|c|}{$1994 / 95$} \\
\hline & & I & $\mathrm{F}$ & I & $\mathrm{F}$ & I & $\mathrm{F}$ & I & $\mathrm{F}$ \\
\hline \multirow{4}{*}{1} & \multirow{2}{*}{ com } & $142,7 \mathrm{a}$ & $242,2 \mathrm{a}$ & $150,9 \mathrm{a}$ & $230,6 \mathrm{a}$ & $174,5 \mathrm{a}$ & $285,1 \mathrm{a}$ & $140,4 \mathrm{a}$ & $217,4 \mathrm{a}$ \\
\hline & & $\pm 10,16$ & $\pm 17,28$ & $\pm 23,95$ & $\pm 24,69$ & $\pm 20,20$ & $\pm 31,53$ & $\pm 14,77$ & $\pm 26,62$ \\
\hline & \multirow{2}{*}{ sem } & $143,9 \mathrm{a}$ & $234,0 \mathrm{~b}$ & $150,6 \mathrm{a}$ & $225,7 \mathrm{~b}$ & $172,1 \mathrm{a}$ & $274,5 \mathrm{~b}$ & $141,6 \mathrm{a}$ & $211,3 \mathrm{~b}$ \\
\hline & & $\pm 7,69$ & $\pm 12,71$ & $\pm 22,16$ & $\pm 30,29$ & $\pm 20,10$ & $\pm 30,60$ & $\pm 16,27$ & $\pm 23,11$ \\
\hline \multirow{4}{*}{2} & \multirow{2}{*}{ com } & $225,9 \mathrm{a}$ & $375,8 \mathrm{a}$ & $237,5 \mathrm{a}$ & $341,6 \mathrm{a}$ & $251,7 \mathrm{a}$ & $380,2 \mathrm{a}$ & $244,5 \mathrm{a}$ & $377,8 \mathrm{a}$ \\
\hline & & $\pm 5,38$ & $\pm 14,35$ & $\pm 7,86$ & $\pm 11,86$ & $\pm 15,48$ & $\pm 19,83$ & $\pm 17,02$ & $\pm 53,06$ \\
\hline & \multirow{2}{*}{ sem } & $221,6 \mathrm{a}$ & $345,5 \mathrm{~b}$ & $237,3 \mathrm{a}$ & $331,0 \mathrm{~b}$ & $252,2 \mathrm{a}$ & $356,8 \mathrm{~b}$ & $244,3 \mathrm{a}$ & $365,4 \mathrm{~b}$ \\
\hline & & $\pm 9,88$ & $\pm 14,06$ & $\pm 7,89$ & $\pm 9,45$ & $\pm 15,17$ & $\pm 20,99$ & $\pm 16,85$ & $\pm 50,88$ \\
\hline \multirow{4}{*}{3} & \multirow{2}{*}{ com } & $315,3 \mathrm{a}$ & $422,2 \mathrm{a}$ & $321,3 \mathrm{a}$ & $432,8 \mathrm{a}$ & $359,7 \mathrm{a}$ & $473,1 \mathrm{a}$ & $327,8 \mathrm{a}$ & $461,4 \mathrm{a}$ \\
\hline & & $\pm 11,92$ & $\pm 25,54$ & $\pm 30,98$ & $\pm 35,93$ & $\pm 21,29$ & $\pm 26,53$ & $\pm 23,55$ & $\pm 31,36$ \\
\hline & \multirow[b]{2}{*}{ sem } & $312,0 \mathrm{a}$ & $402,9 \mathrm{~b}$ & $322,4 \mathrm{a}$ & $426,3 \mathrm{~b}$ & $359,7 \mathrm{a}$ & $464,2 \mathrm{~b}$ & $329,8 \mathrm{a}$ & $451,6 \mathrm{~b}$ \\
\hline & & $\pm 7,82$ & $\pm 12,39$ & $\pm 30,73$ & $\pm 33,44$ & $\pm 20,26$ & $\pm 33,88$ & $\pm 23,90$ & $\pm 30,50$ \\
\hline
\end{tabular}

Valores seguidos por letras diferentes dentro de uma mesma coluna e idade são significativamente diferentes $(\mathrm{P}<0,05)$. Os valores em itálico, referem-se ao Desvio Padrão dos valores médios que os precedem dentro da respectiva coluna. 
segundo delineamento inteiramente casualizado, em esquema fatorial três $\mathrm{x}$ dois (idades dos animais $\mathrm{x}$ tratamentos), considerando-se os períodos de avaliação como repetições do experimento base (primeiro dos quatro períodos de avaliação), de acordo como o modelo:

$$
Y_{i j k l}=\mu+E_{i}+T_{j}+I_{k}+E T_{i j}+E I_{i k}+T I_{j k}+E T I_{i j k}+\varepsilon_{(i j k) l}
$$

Em que: $\mathrm{m}=$ constante geral; $\mathrm{E}_{\mathrm{i}}=$ efeito do experimento ou período de avaliação i (efeito aleatório); $\mathrm{T}$ = efeito do tratamento $\mathrm{j}$ (efeito fixo); $\mathrm{I}_{\mathrm{k}}$ $=$ efeito da idade do animal $\mathrm{k}$ (efeito fixo); $\mathrm{ET}_{\mathrm{ij}}, \mathrm{EI}_{\mathrm{ik}}$, $\mathrm{TI}_{\mathrm{jk}}, \mathrm{ETI}_{\mathrm{ijk}}=$ interações entre os efeitos principais; e, $\mathrm{e}_{(\mathrm{ijk}) 1}=$ erro aleatório, associado a cada observação, pressuposto NID $\left(0, \mathrm{~s}^{2}\right)$.

Os testes "F" realizados na análise de variância para os diferentes efeitos foram estruturados adotando-se $\mathrm{a}=0,05$.

\section{RESULTADOS E DISCUSSÃO}

Observou-se que a mosca-dos-chifres ataca em maior número $(\mathrm{P}<0,05)$ os animais com três anos de idade em comparação com os de dois anos, sendo os de um ano os menos infestados $(\mathrm{p}<0,05)$. O número médio de moscas para o primeiro, segundo, terceiro e quarto anos do estudo foram, respectivamente, cinco, cinco, quatro e cinco, para os grupos com um ano de idade; $15,11,13$ e 27, para os grupos de dois anos e 55, 31, 40 e 51, para os grupos de três anos. Tal observação vem corroborar com o relato de STEELMAN et al. (1996), que sugerem, além do fator idade, que o tamanho dos indivíduos está diretamente relacionado ao número de moscas que o infestam. Os níveis populacionais de $\boldsymbol{H}$. irritans observados podem ser considerados baixos diante dos valores relatados por STEELMAN et al. (1991) e MORRISON \& FOIL (1995). Tal comportamento poderia, talvez, em parte, ser explicado pelas raças bovinas utilizadas em cada caso, bem como, pela abundância de besouros coprófagos observada no local deste estudo.

Na tabela 1, são apresentados os dados de ganho de peso dos animais em estudo, a cada ano, em função dos tratamentos. O ganho médio de peso por animal e por ano foi de nove, cinco, sete e $8 \mathrm{~kg}$ para os grupos com um ano de idade; de 26, 10, 23 e $12 \mathrm{~kg}$ para os grupos de dois anos, e de 16, oito, nove e 11 $\mathrm{kg}$ para os grupos de três anos. Os ganhos médios de peso dos animais tratados com inseticida, em relação aos não tratados, nos quatro anos de estudo, foram 8,$6 ; 16,0$ e $10,4 \%$ superiores $(\mathrm{P}<0,05)$, respectivamente, para animais de um, dois e três anos de idade. Entretanto, quando se consideram apenas as infestações dos grupos de animais não tratados, os ganhos de peso observados $(\mathrm{P}>0,05)$ não podem ser explicados apenas com base na intensidade das respectivas infestações por $\boldsymbol{H}$. irritans.

Na tabela 2, é apresentado o resumo dos efeitos determinados pela Análise de Variância com relação às variáveis empregadas e/ou suas interações. Para o peso vivo final (PVF) foram observados efeitos significativos $(\mathrm{P}<0,05)$ entre os períodos de observação (E), os tratamentos "com" e "sem" inseticidas (T) e a idade dos animais (I), bem como, na interação entre os períodos de observação e a idade dos animais (EI). O efeito sobre as demais interações (ET, TI, ETI) não foram significativos $(\mathrm{P}>0,05)$. Quadro semelhante foi verificado para o ganho médio diário de peso (GMD), com exceção da variável idade dos animais, que apresentou efeito não significativo (P>0,05).

Contrariando os relatos de SCHREIBER et al. (1987) e MORRISON \& FOIL (1995), que não encontraram danos ocasionados pela ação de $\boldsymbol{H}$. irritans nos animais das raças por eles estudadas, os resultados aqui obtidos permitem concluir que, para bovinos machos da raça Nelore, mesmo com as reduzidas infestações observadas, a mosca-dos-chifres causa prejuízos e a sua presença ou ausência é mais importante do que a intensidade de infestação. Sugerese que, por tratar-se de um problema recente, visto que este parasito aqui se estabeleceu a partir de 1991 (BIANCHIN \& ALVES, 2002), os animais ainda não

Tabela 2 - Efeitos da mosca-dos-chifres sobre o ganho de peso final (PVF) e ganho médio diário (GMD) em bovinos nelores, determinada pela Análise de Variância, segundo delineamento inteiramente casualizado, em esquema fatorial 3 x 2 (idades dos animais "I" $\mathrm{x}$ tratamentos "T"), considerando-se os períodos de avaliação "E" como repetições do experimento base (primeiro dos quatro períodos de avaliação), durante quatro estações chuvosas (outubro a maio) consecutivas, de 1991 a 1995, em Campo Grande, MS.

\begin{tabular}{lccc}
\hline FV & GL & PVF & GMD \\
\hline E & 3 & $*$ & $*$ \\
T & 1 & $*$ & $*$ \\
I & 2 & $*$ & $n s$ \\
ET & 3 & $n s$ & $n s$ \\
EI & 6 & $*$ & $*$ \\
TI & 2 & $n s$ & $n s$ \\
ETI & 6 & $\mathrm{~ns}$ & $\mathrm{~ns}$ \\
QMresíduo & 270 & 821.103 & 0.01001 \\
\hline
\end{tabular}

Ns $=$ não-significativo $*=$ significativo a $5 \%$ de probabilidade 
tolerem a simples presença da mosca. Entretanto, espera-se que, após um período mais longo de convivência, os animais venham a suportar infestações, como as atualmente verificadas, apresentando prejuízos de menor relevância.

Frente aos dados atualmente disponíveis, para a região dos Cerrados de Mato Grosso de Sul, verifica-se que os danos ocasionados por $\boldsymbol{H}$. irritans em animais da raça Nelore são menores do que a previsão feita por HONER et al. (1991). Mesmo assim, representam um prejuízo de US\$ 150 milhões para o país, conforme a estimativa feita por GRISI et al. (2002), necessitando, por isso, ser mantida sob controle.

Foi verificado por BARROS (2001), no Pantanal sul-mato-grossense, que o número médio de mosca-dos-chifres por vaca da raça Nelore excedeu a 85 durante a ocorrência dos picos populacionais, mantendo-se abaixo de 35 moscas/vaca durante a maior parte do restante do período. OLIVEIRA \& FREITAS (1997) verificaram em vacas leiteiras de quatro propriedades, nas quais a mosca-dos-chifres não era alvo de controle específico (havia controle de carrapatos e endoparasitos), que a carga de insetos nos meses de maior infestação (outubro a março) foi de 15,7 $\pm 36,4$ moscas/animal. Os níveis populacionais de $\boldsymbol{H}$. irritans verificados por estes autores, encontramse muito próximos aos aqui observados, sendo muito inferiores às 200 moscas/animal freqüentemente referidos nos EUA (STEELMAN et al., 1991; MORRISON \& FOIL, 1995). Entretanto, sabe-se que há produtos utilizados no controle de outros parasitos bovinos que também agem sobre $\boldsymbol{H}$. irritans. Além disso, o controle químico convencional, a exemplo do que ocorre em outros países, se não empregado com a devida racionalidade e visando a todos os parasitos presentes de um modo integrado, conduz rapidamente à seleção de populações resistentes. Há registro de que a mosca-dos-chifres já está menos sensível à maioria dos produtos em uso do que quando da sua entrada no País (BARROS et al. 2002). Diante desta situação, é possível que, de modo geral, os níveis populacionais de $\boldsymbol{H}$. irritans tenderão a aumentar, pela elevação gradual da dificuldade de seu controle.

Outro fator com potencial para aumentar os níveis populacionais e/ou os danos da mosca-doschifres na região é a introdução cada vez maior de animais $\boldsymbol{B}$. taurus ou seus cruzamentos, mais sensíveis à referida mosca (DRUMMOND et al., 1981; HONER et al., 1991), bem como a outros parasitos, entre os quais o carrapato B. microplus (UTECH et al., 1978; GOMES et al., 1989), do que os animais da raça Nelore.

\section{CONCLUSÃO}

Concluiu-se que a presença da mosca-doschifres, ainda que em baixos níveis populacionais, em bovinos machos da raça Nelore com um, dois, três anos de idade, representou redução significativa $(\mathrm{P}<0,05)$ no ganho de peso dos animais não tratados $\mathrm{e}$ expostos ao parasito.

\section{REFERÊNCIAS BIBLIOGRÁFICAS}

BARROS, A.T.M. Dynamics of Haematobia irritans irritans (Diptera: Muscidae) infestation on Nellore cattle in the Pantanal, Brazil. Memórias do Instituto Oswaldo Cruz, Rio de Janeiro v.96, n.4, p.445-450, 2001.

BARROS, A.T.M. et al. Susceptibility to diazinon in populations of the horn fly, Haematobia irritans (Diptera: Muscidae), in Central Brazil. Memórias do Instituto Oswaldo Cruz, v.97, n.6, p.905-907, 2002.

BIANCHIN, I.; ALVES, R.G.O. Mosca-dos-chifres, Haematobia irritans: comportamento e danos em vacas e bezerros Nelore antes da desmama. Pesquisa Veterinária Brasileira, v.22, n.3, p.109113, 2002.

BYFORD, R.L.; CRAIG, M.E.; CROSBY, B.L. A review of ectoparasites and their effect on cattle production. Journal of Animal Science, v.70, n.2, p.597-602, 1992.

COCKE Jr., J.; KNUTSON, R.; LUNT, D.K. Effects of horn fly control with lambda cyhalothrin ear tags on weight gains in weaning calves in Texas. Southwestern Entomologist, v.14, n.4, p.357-362, 1989.

DRUMMOND, R.O. et al. Estimated losses of livestock to pests. In: PIMENTEL, D. CRC handbook of pest management in agriculture. Boca Raton : CRC, 1991. V.1, p.317-346.

GOMES, A. et al. Populations of the cattle tick Boophilus microplus on purebred Nellore, Ibage and Nellore x European crossbreeds in the Brazilian Savanna. Tropical Animal Health and Production, v.21, n.1, p.20-24, 1989.

GRISI, L. et al. Impacto econômico das principais ectoparasitoses em bovinos no Brasil. A Hora Veterinária, v.21, n.1, p.8-10, 2002.

HOGSETTE, J.A.; PRICHARD, D.L.; RUFF, J.P. Economic effects of horn fly (Diptera: Muscidae) populations on beef cattle exposed to three pesticide treatment regimes. Journal of Economic Entomology, v.8, n.4, p.1270-1274, 1991.

HONER, M.R.; BIANCHIN, I.; GOMES, A. Mosca-dos-chifres: histórico; biologia e controle. Campo Grande : EMBRAPACNPGC, 1991. 34p. (EMBRAPA-CNPGC. Documentos, 45).

KUNZ, S.E. et al. Estimated losses of livestock to pests. In: PIMENTEL, D. CRC handbook of pest management in agriculture. Boca Raton : CRC, 1991. V.1, p.69-98.

KÖPPEN, W. Klimakarte der erde. Goth a: Perthes, 1928. n.i.

LIMA, L.G.F.; PRADO, A.P.; PERRI, S.H.V. Comparison of two methods (visual estimates and filming) for counts of horn flies 
(Haematobia irritans irritans) (L.) (Diptera: Muscidae). Veterinary Parasitology, v.103, n.3, p.227-235, 2002.

MORRISON, D.G.; FOIL, L.D. Effect of horn fly (Diptera: Muscidae) control during the spring on calf production by fallcalving beef cows. Journal of Economic Entomology, v.88, n.1, p.81-84, 1995.

OLIVEIRA, G.P.; FREITAS, A.R. Comportamento da Haematobia irritans em fazendas com diferentes manejos de bovinos. Ciência Rural, v.27, n.2, p.279-284, 1997.

SANTOS, R. A raça Nelore e Nelore mocho. In: SANTOS, A.R. $O$ zebu: edição comemorativa dos 60 anos do registro genealógico da ABCZ. Uberaba : Agropecuária Tropical, 1998. p.251-292.

SAS Institute Inc. SAS User's guide: statistics. Version 6.12. Cary : SAS Institute, 1998. 584p.
SCHEIBER, E.T. et al. Effects of horn fly (Diptera: Muscidae) control on cows and gastrointestinal worm (Nematode: Trichostrongylidae) treatment for calves on cow and calf weight gains. Journal of Economic Entomology, v.80, n.2, 451-454, 1987.

STEELMAN, C.D. et al. Interactive response of the horn fly (Diptera: Muscidae) and selected breeds of beef cattle. Journal of Economic Entomology, v.84, n.4, p.1275-1282, 1991.

STEELMAN, C.D. et al. The effects of selection for size in cattle on horn fly population density. Medical and Veterinary Entomology, v.10, n.2, p129-136, 1996.

UTECH, K.W.B.; WHARTON, R.H.; KERR, J.D. Resistance to Boophilus microplus (Canestrini) in different breeds of cattle. Australian Journal of Agricultural Research, v.29, n.4, p.885$895,1978$. 